\title{
Neuropathological Sequelae of Long-Term Allogeneic and Syngeneic Neural Transplantation into the Hippocampus
}

\author{
Sanjay N. Patel ${ }^{1,2, *}$, Tim R. Kershaw ${ }^{2}$, John Williams ${ }^{1}$, Jeffrey A.Gray ${ }^{2}$, Peter L. Lantos ${ }^{1}$ and John D. Sinden ${ }^{2}$ \\ The Institute of Psychiatry, Departments of Neuropathology ${ }^{I}$ and Psychology ${ }^{2}$, DeCrespigny Park, London \\ SE5 8AF, U.K. *Present address: University of Oxford, Department of Zoology, South Parks Road, Oxford, \\ OX1 3PS, U.K.
}

\section{SUMMARY}

The long-term fate of multiple intrahippocampal allogeneic transplants of fetal basal forebrain tissue was studied in neonatally tolerised and immunised groups of rats with lesions of the fimbria-fornix. Despite the good survival of the allografts in all groups, unexpected transplant-associated host hippocampal neuropathology was discovered 12 months after transplantation, which consisted of (i) CA1 cell degeneration and (ii) abnormal accumulations of phosphorylated neurofilaments in neuronal perikarya and axonal swellings only within the host hippocampal neuropil and not of the transplanted tissue. This neurofilament abnormality, identified by RT97 immunohistochemistry, was significantly greater in the transplanted rats compared to the non-grafted lesion-only and sham-lesioned rats $(p<0.01)$. The same type of neurofilament abnormality was again observed in a second, separate experiment using unilateral and bilateral syngeneic and allogeneic transplants. The neuropathology was significantly $(p<0.05)$ greater in the transplanted side of the unilateral transplanted rats compared to the non-transplanted lesion-only control side of the same animals, showing that transplantation per se was a major factor involved in the pathogenesis of this neuropathology, irrespective of the type of transplant

Reprint address:

Sanjay N. Patel

The University of Oxford

Department of Zoology

South Parks Road

Oxford OX1 3PS

U.K. (syngeneic or allogeneic). In addition, a small degree of neurofilament abnormality was also found within the transplants in the second experiment, but not in the first. The results show that, under certain conditions, specific local neuropathological damage to the surrounding host neural tissue can develop in long-surviving allografted and syngrafted animals.

\section{KEY WORDS}

allograft, hippocampus, neurofilament, immunological tolerance, neuropathology, immunisation, rat

\section{INTRODUCTION}

Neural tissue transplantation into the central nervous system has been successful in reversing a variety of lesion-induced functional deficits in animals and has already been applied as a treatment strategy for Parkinson's disease in man $/ 3,10,22$, 29/. The transection of the fimbria-fornix, denervating the hippocampus of transmitter-specific subcortical inputs including cholinergic fibres from the medial septal area, has been shown to result in a significant loss in spatial memory performance in a variety of maze tasks, which can be reversed by the transplantation of cholinergic-rich fetal tissue into the hippocampus $/ 10,29 /$. Despite the relative immunological privilege of the brain $/ 5 /$, intracerebral neural allografts can undergo spontaneous $/ 23 /$, and induced $/ 13 /$, immunological rejection, and this may lead to a loss in any cognitive improvements the transplants may have had.

In the first experiment described in this report, we studied the possibility of reducing the risk of 
spontaneous rejection of allogeneic transplants placed into the denervated hippocampus, by inducing immunological tolerance in the host animals (F344 rats) to the donor tissue (from PVG rats). Immunological tolerance is a well recognised phenomenon whereby the host is rendered immunologically unresponsive to foreign donor tissue which differs from the host in major transplantation antigens. One method (and the one used here) of achieving this is by injecting donor tissue antigens into the newborn host animal $/ 2 /$. Host immunisation was performed eleven months after transplantation as a test to examine whether tolerance can be broken and whether intracerebral transplant rejection can be provoked.

However, unexpected transplant-associated host hippocampal neuropathology was also discovered upon histological examination of the rats 12 months after transplantation in all but one of the transplanted rats. This surprising, and previously unpublished, result was subsequently explored further in the second experiment.

\section{METHODS}

\section{Experiment 1}

Inbred Fisher F344 (RT-1 ${ }^{\text {lv }}$ ) rats (used as hosts) and inbred PVG (RT-1 ${ }^{c}$ ) rats (used as donors), differing in major histocompatibility complex (MHC) class I and II, were obtained from HarlanOlac, U.K.

Six time-mated female Fisher F344 rats were allowed to give birth and the rat pups of three of the litters were given intraperitoneal injections of gamma-irradiated (at 2,500 rads from a caesium source) PVG splenocytes, within the first $48 \mathrm{~h}$ of birth (referred to hereafter as "tolerised" rats). The pups from the remaining three litters were left uninjected and therefore served as age-matched "non-tolerised" controls. The splenocytes were prepared by removing the spleens from adult PVG rats under deep terminal anaesthesia and the tissue was minced into a suspension which was then filtered through a sterile gauze. The red blood cells were removed by incubation for $1 \mathrm{~min}$ in $0.15 \mathrm{M}$ ammonium chloride followed by washing (3 times) in Hank's Balanced Salt Solution (HBSS from
GIBCO, U.K.) and re-suspended in HBSS. Each "tolerised" pup received $100 \mu 1$ of $1 \times 10^{7}$ cells in HBSS. Only the male rats were used for the subsequent lesioning and transplantation experiment (the female rats were also transplanted, but these results will be presented in a separate paper).

Fimbria-fornix and sham lesions were performed three months after tolerisation under sterile conditions with the rats under deep equithesin anaesthesia $(0.3 \mathrm{ml} / 100 \mathrm{~g})$ using the procedure of Feldon et al. /12/. With lambda and bregma in the same horizontal plane, the forceps (with tips 1.5 $\mathrm{mm}$ apart) was inserted at $1.0 \mathrm{~mm}$ posterior to bregma, with its lower tip $5.3 \mathrm{~mm}$ below bregma. The fimbria-fornix lesion was produced by moving the forceps into the contralateral hemisphere 4.0 $\mathrm{mm}$ across the midline and clamping the forceps shut for $2 \mathrm{~min}$ prior to opening and retraction. In sham-lesioned rats, the forceps was introduced only $2.0 \mathrm{~mm}$ into the ipsilateral cortex prior to clamping, opening and retraction.

Two-three weeks after lesioning, the rats received multiple PVG fetal (E14-15) basal forebrain tissue transplants into the hippocampus. All procedures were performed under aseptic conditions according to the methods of Björklund $e t$ al. /4/. Briefly, foetuses were removed from timemated females under deep pentobarbitone anaesthesia by laporotomy and E14-15 basal forebrain tissue was collected in sterile $0.6 \%$ glucose $/ 0.9 \%$ saline (GS), dissociated with sterile $0.1 \%$ trypsin (from Sigma) in GS for $20 \mathrm{~min}$ at $37^{\circ} \mathrm{C}$, washed 4 times in sterile GS, further dissociated by gentle trituration and re-suspended in GS to a volume equivalent to $10 \mu \mathrm{l}$ per dissected tissue piece. Two $\mu l$ of the cell suspension (containing $1-2 \times 10^{5}$ cells) were taken up into a 10 $\mu l$ Hamilton syringe and injected at the following (bilateral) co-ordinates: $\mathrm{AP}=-\mathbf{3 . 3}$ with $\mathrm{L}= \pm 1.5$ and $\mathrm{V}=-3.8$; and $\mathrm{AP}=-4.3$ with $\mathrm{L}= \pm 2.2$ and $\mathrm{V}=$ -3.4. The injection was performed over $2 \mathrm{~min}$ and the pipette was left in situ for a further 2 min before withdrawal.

Host immunisation was performed 11 months after transplantation and consisted of four injections (one per week for four weeks) of gamma-irradiated PVG splenocytes $\left(10^{7}\right.$ cells per rat in $100 \mu \mathrm{l}$, subcutaneously; prepared as above) in HBSS containing Freund's Incomplete Adjuvant (Miles 
Lab Inc.) (immunised rats). The non-immunised rats did not receive any injections. Twelve months after transplantation, the rats were perfused through the left ventricle of the heart (and into the aorta), under deep pentobarbitone anaesthesia with sodium phosphate buffered saline (PBS, $20 \mathrm{ml}, \mathrm{pH} 7.4$ ) using a Watson-Marlow peristaltic pump (\#205S). This was immediately followed by $200 \mathrm{ml}$ of $4 \%$ paraformaldehyde in phosphate buffer (PB, $\mathrm{pH} 7.4)$ perfused over a 6-8 min period. The brains were removed and kept in the fixative for 1-2 $\mathrm{h}$, washed in PBS and kept in 25\% sucrose in PB until the brains sank prior to cryostat sectioning at $20 \mu \mathrm{m}$. Serial sections were collected in series on gelatin coated slides for histological staining for Nissl, acetylcholinesterase (ACHE; according to the method of El Badawi and Shenk /11/), and Bielschowsky silver staining (according to the method specified in Cox $/ 6 /$ ); and for immunohistochemical labelling with antibodies to PVG MHC class I (YR5/12, rat monoclonal), MHC class II (OX6, mouse monoclonal) (both from Serotec) and RT97 (mouse monoclonal, supernatant /1/) (gift from Prof. B.H. Anderton) using standard avidinbiotin protocols (DAKO). The RT97 antibody was used to study possible cytoskeletal changes in transplanted neurons, which has been reported previously $/ 8,9 /$. Primary antibodies were used at a dilution of 1:100 in PBS. For RT97, the nonspecific background staining was blocked by pretreatment of the sections with methanol containing $1 \% \mathrm{H}_{2} \mathrm{O}_{2}$ and after washing in buffer, by incubation for $30 \mathrm{~min}$ in $10 \%$ normal lamb serum. The chromogen for RT97 labelling consisted of 0.05\% 3,3-diaminobenzidine tetrahydrochloride with $0.03 \% \mathrm{H}_{2} \mathrm{O}_{2}$ in phosphate buffered saline. Visualisation for the OX6 and YR/12 antibodies was performed with a red alkaline phosphatase kit (Vector labs). The sections were counterstained with Harris's haematoxylin, dehydrated and mounted in Eukitt.

The YR5/12 and OX6 antibodies were also tested on normal F344 and PVG rat brain, kidney and spleen tissue, giving expected results. Control sections, without the primary antibodies, but with the respective second and third layers showed no immunoreactivity. The RT97 staining of the grafted tissue was repeated twice, but using different batches of the RT97 antiserum to rule out the possibility of a peculiarity of the staining which may be related to the particular batch of antiserum. Similar staining was obtained with both batches.

The extent of neurofilament abnormality (see below) in the various groups of rats was estimated by quantifying the frequency of RT97 positive structures (cell bodies and axonal spheroids) within the hippocampi (both hemispheres) of the 9 (of 10) grafted and 8 non-grafted (4 sham-lesioned and 4 lesioned-only) rats from the sample of RT97 labelled sections and expressed as a mean number of labelled structures per section per rat (to control for the different number of sections examined in each of the rats; see Table 1). The counting was performed "blind", i.e., without reference to the tolerisation, immunisation and control status of the animals. Statistical analyses were performed with the MannWhitney $U$ test and photographs were taken using a Zeiss photomicroscope. The amount of CAl cell damage observed in three of the transplanted rats was also approximately quantified in 12-15 Nissl

TABLE 1

Mean number of RT97 profiles per section per rat in each group of experiment 1

\begin{tabular}{|c|c|c|c|c|c|c|c|c||}
\hline \multirow{2}{*}{} & \multicolumn{2}{|c|}{$T$ and I } & \multicolumn{2}{c|}{$T$ and NI } & \multicolumn{2}{c|}{ NT and I } & \multicolumn{2}{c|}{ NT and NI } \\
\cline { 2 - 9 } & G & NG & G & NG & G & NG & G & NG \\
\hline MEAN & 2.798 & 0.125 & 0.535 & 0.14 & 0.8 & 0.1 & 0.3 & 0.2 \\
sem & 1.73 & 0.09 & 0.095 & 0.024 & 0 & 0 & 0 & 0 \\
N & 4 & 2 & 2 & 3 & 1 & 2 & 2 & 1 \\
no.sects & 59 & 15 & 23 & 27 & 15 & 15 & 22 & 5 \\
\hline
\end{tabular}

The treatment groups were: $\mathrm{T}$ and $\mathrm{I}=$ tolerised and immunised; $\mathrm{T}$ and $\mathrm{NI}=$ tolerised and non-immunised; $\mathrm{NT}$ and $\mathrm{I}=$ nontolerised and immunised; NT and NI = non-tolerised and non-immunised. G=grafted, NG=non-grafted. 
stained sections taken throughout the dorsal hippocampus, containing the transplant. A camera lucida attachment to the microscope was used to draw and measure the region of normal and damaged CA1 cell layer on a sheet of paper. An image analysis system, Image 1.92 for the Macintosh, was used to measure the normal and damaged CAl cell regions. The damage was then expressed as the percentage of the CA1 cell region damaged over the whole CA1 cell region measured in the 12-15 sections (bilaterally).

\section{Experiment 2}

The second experiment was designed to determine whether or not the observed transplant associated host damage was due specifically to the allogeneic nature of the transplants or whether it was simply due to the presence of a cell suspension transplant per se. This was achieved by (i) comparing any neuropathological effects of allografts with those of syngeneic cell suspension transplants; (ii) using unilateral syngeneic and allogeneic transplants, in which the contralateral non-transplanted hippocampi served as a within animal lesion-only control side to which the transplanted side could be compared; and (iii) using bilateral syngeneic and 'mixed' transplants (where allografts were placed into one hippocampal side and syngrafts into the other). The second experiment also served to determine whether the transplant associated host neurofilament abnormality could be replicated. If the observed RT97 positive abnormality was unique to the first experiment (and unrelated to transplantation), then similar damage of the host should not be found in this second experiment. The rats were not tolerised or immunised, so that only the effects of transplantation could be observed.

Four unilateral and four bilateral grafted F344 rats, having a total of 8 syngeneic and 4 allogeneic transplants, were used for this experiment. Of the unilateral grafted rats, two had syngeneic transplants and two had allogeneic transplants intc one hippocampal side. Two rats had bilateral syngeneic transplants; and a further two rats had mixed transplants, with a syngeneic graft into either the left or right hippocampus and an allograft into the contralateral hippocampus. The bilateral trans- planted rats allowed a direct comparison of the local effects of syngrafts and allografts upon the host hippocampus and therefore an assessment of the neuropathological effects of the type of transplant (syngeneic or allogeneic). Finally, one rat served as a non-grafted, lesion only control (one of the rats allocated to be controls died).

The embryonic basal forebrain tissue for the syngeneic transplants was taken from F344 rat fetuses and for allogeneic transplants, from PVG rat fetuses, both prepared as described above in experiment 1 . In contrast to the first experiment, only single allogeneic or syngeneic cell suspension transplants were placed into the hippocampus of fimbria-fornix lesioned rats, at the anterior transplantation site given above.

With the exception of neonatal tolerisation and immunisation (which were not employed), all of the procedures used in experiment 1 were repeated in this second experiment. The rats were perfused 11 months after transplantation, to coincide with the time that the immunisation injections were performed in experiment 1. The histological analyses consisted of Nissl and ACHE staining and immunohistochemical labelling with the RT97 antibody (as described above). The counting of RT97 positive profiles (in 18-23 sections per brain) was performed "blind" regarding the type of the transplant. The paired t-test was used for statistical analyses of the unilateral transplanted rats.

\section{RESULTS}

\section{Experiment 1}

All of the grafted rats, tolerised and nontolerised, which were either immunised or not, had surviving and healthy, intrahippocampal allografts. The transplants were generally placed well within the dorsal hippocampus (Figs. 1,2), and in some cases also partly within the fimbria. The allografts showed no histological evidence of immunological rejection: there were no lymphocytic infiltrations, little macrophagic activity and little or no MHC class I or II staining. In addition, the health of the allografts was indicated by Bielschowsky silver staining which showed no degenerating neurons within the transplants, but did reveal argyrophilic, presumably degenerating, CAl cells in the vicinity 

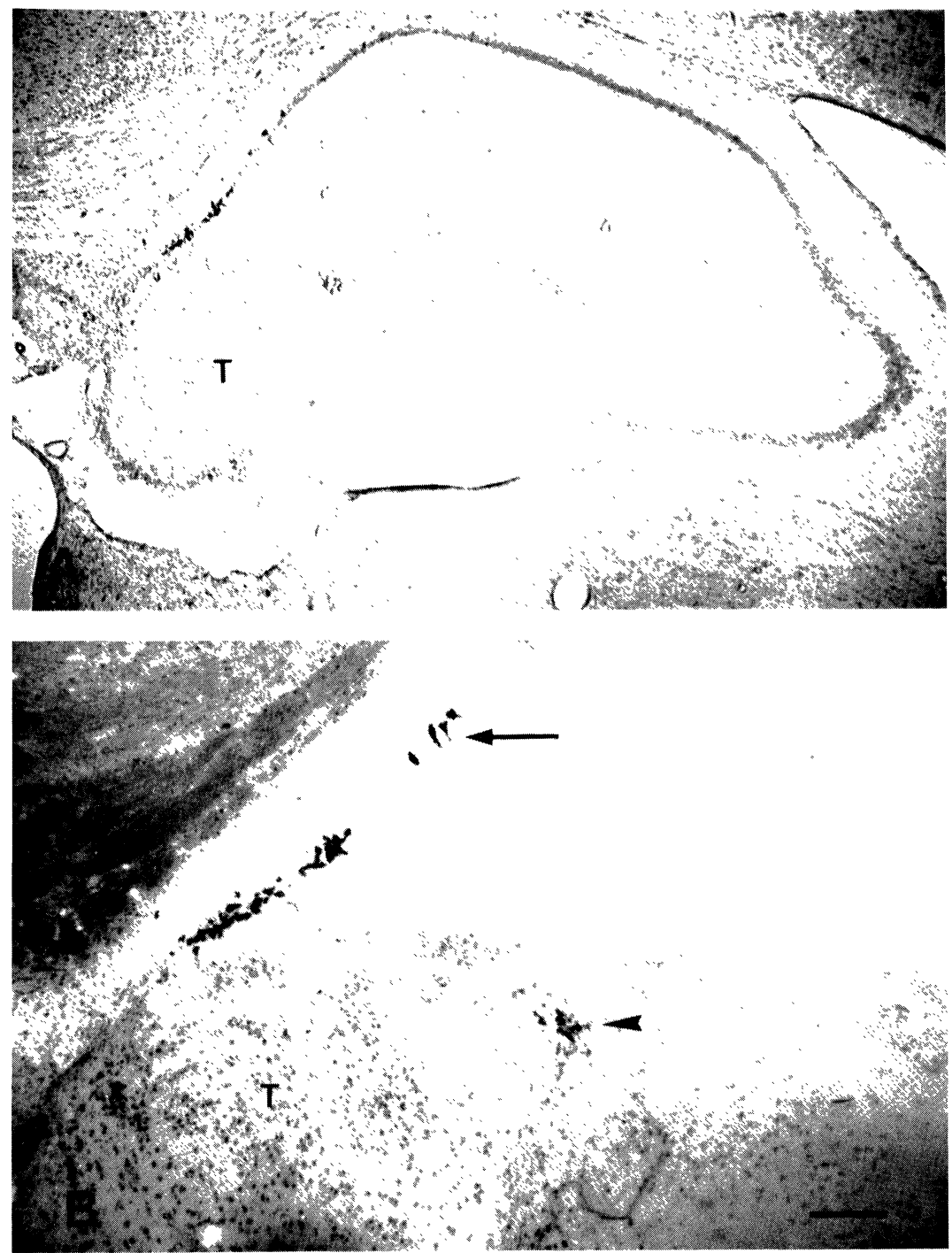

Fig. 1: [A] Nissl stained section of a healthy transplant $(\mathrm{T})$ within the hippocampus showing host CA1 cell degeneration, which is more clearly shown in a Bielshowsky stained preparation (arrow in [B], damaged cells appear black). There is also a small necrotic area at the edge of the transplant composed of dying host dentate gyrus granule cells or cells of the transplant (arrowhead). No other neuritic changes were observed in Bielschowsky stained preparations. Scale bar $=100$ $\mu \mathrm{m}$.

of the transplants in three of the ten transplanted rats in which the transplants were located close to the CA1 cell layer (Fig. 1). Approximately 20\%, $23 \%$ and $12 \%$ of the CAl cell layer of the hippocampus was damaged in these three transplanted rats. CA1 cell damage was not found in the sham lesioned and lesioned-only groups of rats.

No abnormal RT97 staining was found within the transplants and few RT97 positive processes could be seen traversing from the graft into the host (Fig. 2). Of the 9 (out of 10) transplanted rats examined with RT97 immunohistochemistry, all but one had abnormal labelling of perikarya and axonal swellings (or spheroids) to varying degrees, within cells of the host hippocampal tissue surrounding the allografts, but not within the allograft tissue itself (Fig. 2A-F). These abnormal profiles were present predominantly within the axonal and dendritic fields 

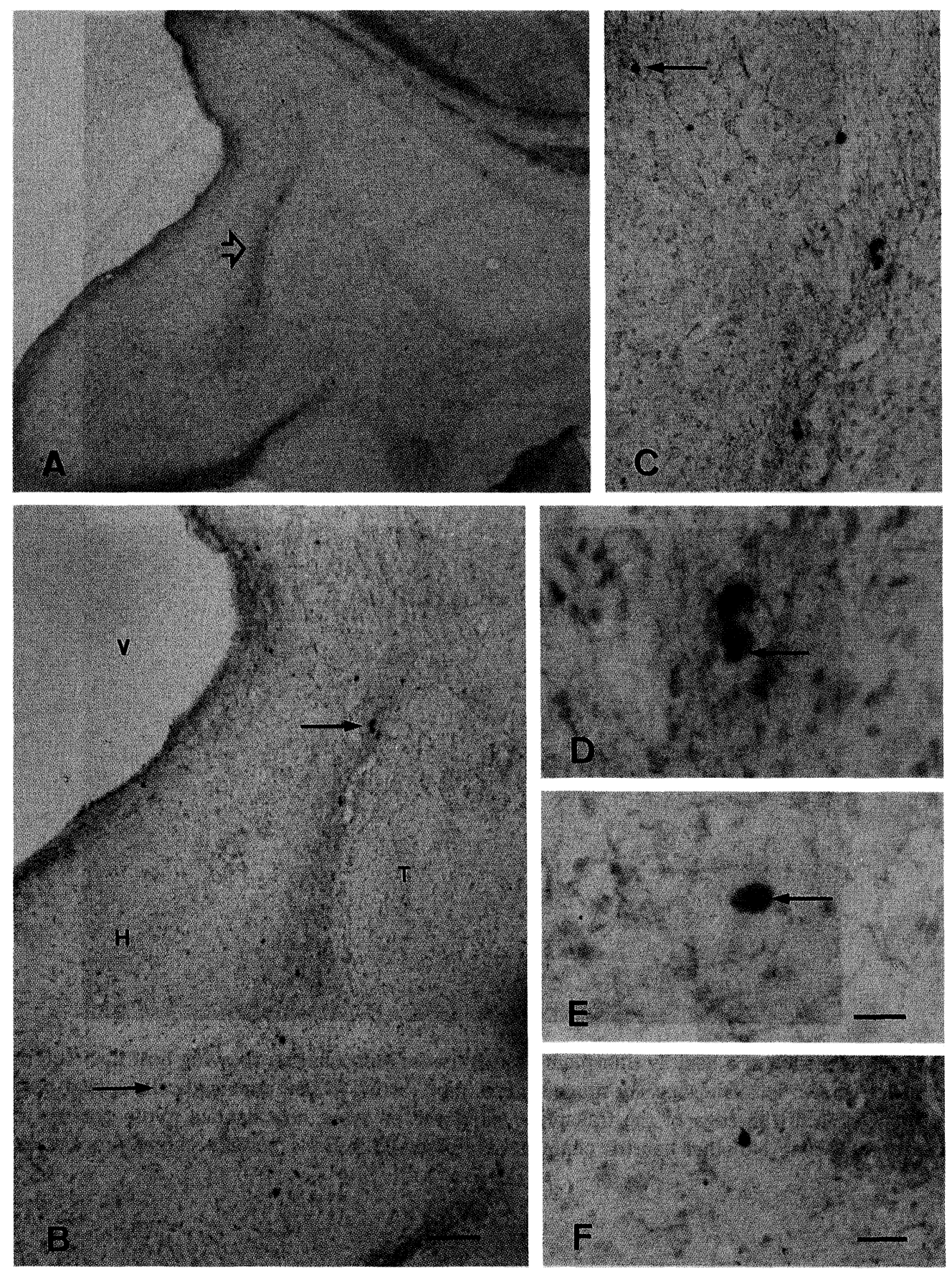

Fig. 2: RT97 immunohistochemical staining (appears brown) of allografted tissue showing abnormal labelling of neuronal perikarya and axonal spheroids only within the host hippocampal tissue surrounding the transplant (which is located in the centre of the hippocampus; arrow in A). A high power view of the transplant (T) is shown in B. Arrows depict presumed RT97 positive neuronal cell bodies in the host hippocampus $(\mathrm{H})$, which are shown in higher power in $\mathbf{C}$, D, and E; note that the transplant itself has little RT97 labelling, normal or abnormal. [C] The RT97 positive axonal spheroids were sometimes found to resemble axonal retraction balls with an axonal tail (arrow). In other cases [D and E], presumed nuclei could be clearly seen within the RT97 positive neuronal perikarya (arrows). Some of the staining was also flame-like in appearance [F]. The sections were counterstained with haematoxylin. $V=$ ventricle. Scale bars: $B$ $=124 \mu \mathrm{m} ; \mathrm{C}$ and $\mathrm{F}=53 \mu \mathrm{m} ; \mathrm{D}$ and $\mathrm{E}=21 \mu \mathrm{m}$. 
of the hippocampus and not within the pyramidal cell layers, indicating that the RT97 positive perikarya were probably of interneurons or nonpyramidal projection neurons. The number of RT97 positive structures was significantly greater in the transplanted hippocampi compared with the nontransplanted sham-lesioned and lesion-only hippocampi ( $<0.01 ; U=7.5 ; z=2.74)$ (Fig. 3). Fimbriafornix lesioned (non-grafted) rats had very few such RT97 positive profiles (which were of axonal spheroids and not of perikarya). No significant differences in this neurofilament abnormality were found either between tolerised and non-tolerised rats or between the immunised and non-immunised rats, although the greatest incidence and the most variability was found in the transplanted rats which were both tolerised and immunised (Table 1). No abnormal staining was found in any other brain region examined (including the medial septum).

\section{Experiment 2}

All of the allogeneic and syngeneic transplants had survived and were located either within the upper blade of the dentate gyrus or in the space between the hippocampus and the stria medullaris (and sometimes extending into the third ventricle). The transplants were healthy, but were smaller than those in the first experiment, presumably because these rats received a single injection of the cell suspension. Some macrophagic activity was observed around small areas of the transplants in the hippocampus. There was no CA1 cell damage found in any of the animals in this experiment, probably because none of the transplants was located close to the CA1 cell layer.

Numerous abnormal RT97 positive neuronal perikarya and axonal swellings were found in the 12 hippocampi with transplants and only one abnormal structure was found in the 4 non-transplanted lesion-only control hippocampal sides of the unilateral grafted rats and no abnormal staining was present in the single non-transplanted lesion-only control rat (Fig. 4). There was a significantly greater frequency of abnormal RT97 positive profiles within the transplanted hippocampi of the unilateral grafted rats compared to the nontransplanted, contralateral control side of the same animals (paired $t=3.48, d f=3, p<0.05$ ) (Fig. 5). The greatest number of abnormal RT97 positive profiles were found in the hippocampi of the two mixed transplanted rats; fewer abnormal RT97 positive profiles were present within the two bilateral syngeneic transplanted rats, but these were still greater than in the controls (Fig. 5). The host neurofilament abnormality was found in the hippocampi containing either syngeneic or allo-

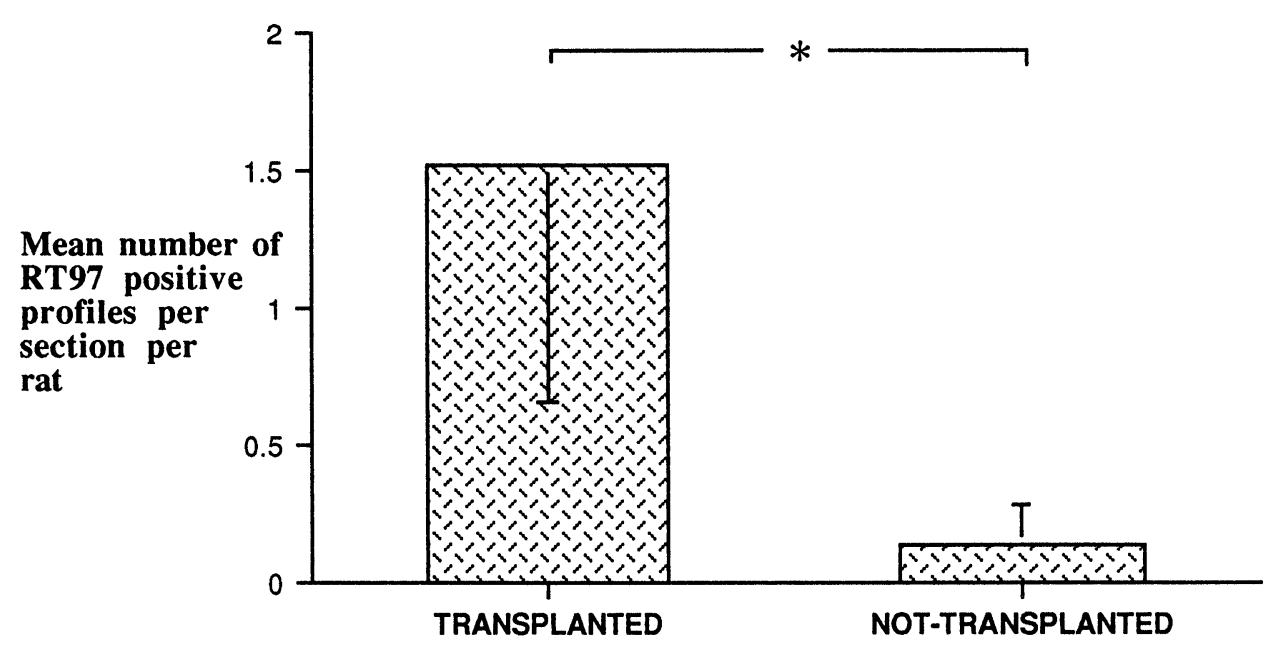

Fig. 3: The graph shows the mean frequency of RT97 postive profiles (showing a single standard error of the mean) encountered within the host hippocampi of the 9 grafted and 8 non-grafted groups of rats from experiment 1 . A significantly greater incidence of RT97 positive profiles was found in the grafted group compared to non-grafted (lesion-only and sham lesion) group $(* \mathrm{p}<0.01)$. 


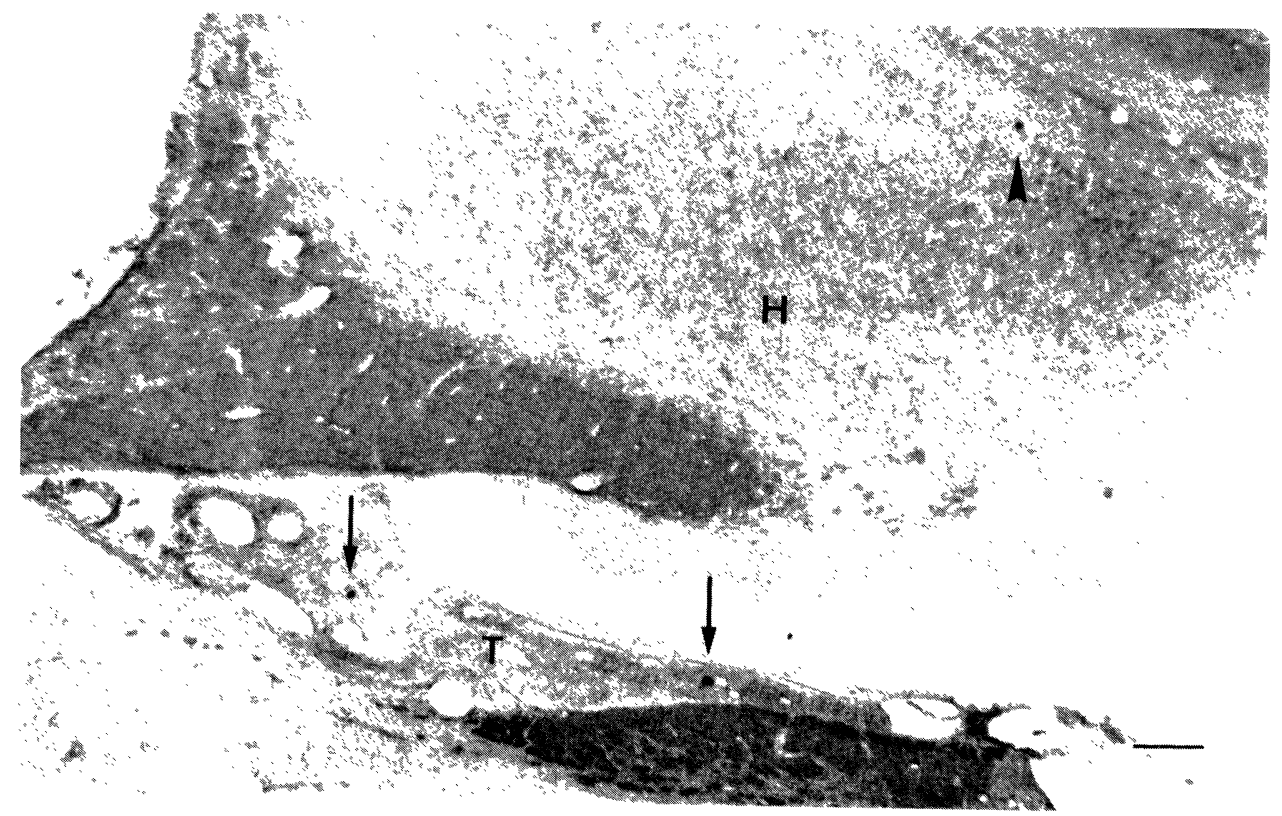

Fig. 4: Photograph of a transplant (T) located below the hippocampus of a rat from experiment 2 . RT97 positive neuronal perikarya are seen both within the transplant (arrow) and in the surrounding host hippocampus (H) (arrowhead) Haematoxylin counterstained. DG = dentate gyrus. Scale bar $124 \mu \mathrm{m}$.

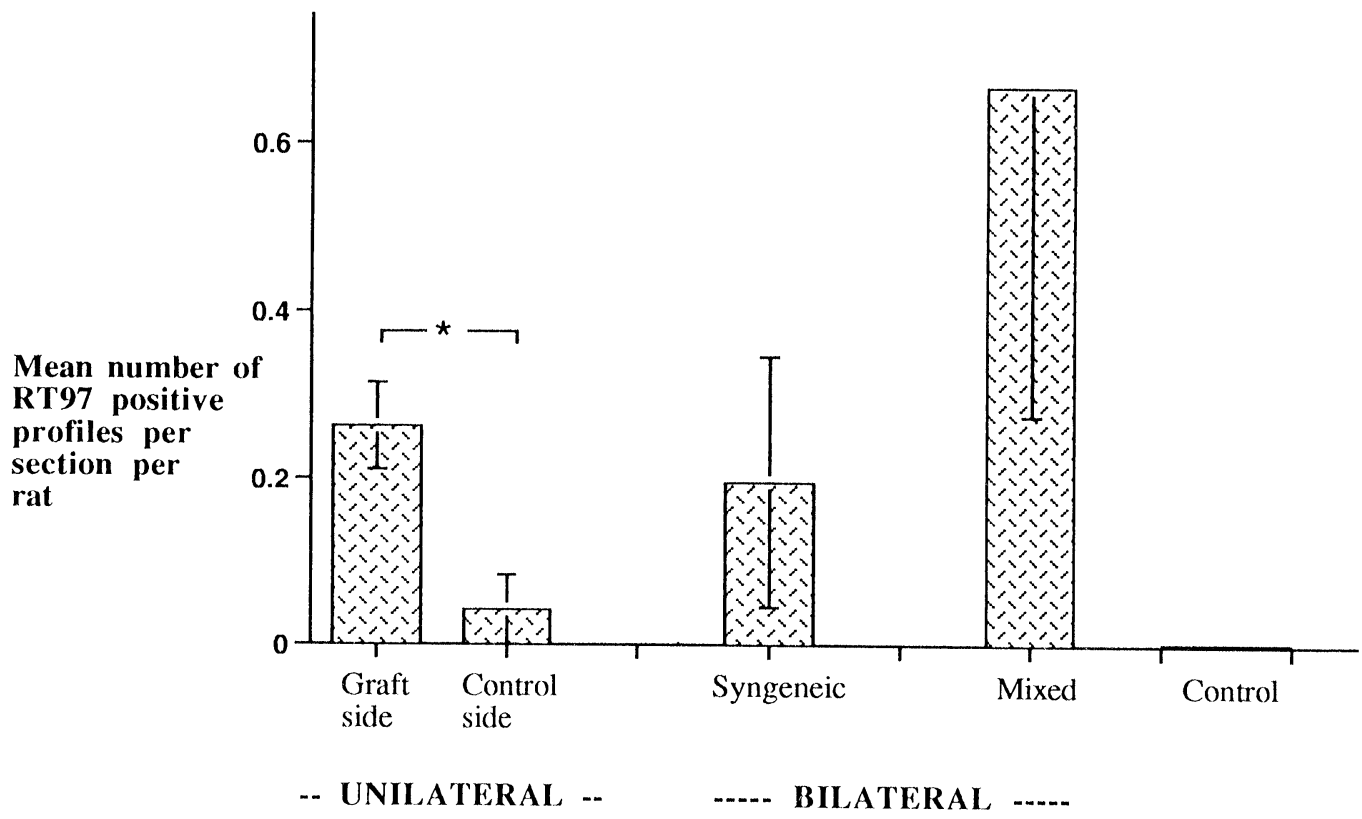

Fig. 5: The mean number of RT97 positive profiles (with standard errors of the mean) present within the grafted and nongrafted denervated hippocampi in the different bilateral and unilateral syngeneic and allogeneic groups of rats from experiment 2. The left part of the graph shows the number of RT97 positive profiles in the unilateral syngeneic and allogeneic groups together: the number of profiles is significantly greater in the grafted side compared to the non-grafted side in the same animals $\left({ }^{*} p<0.05\right)$. The right part of the graph shows the average number of profiles in the bilateral syngeneic only group and the mixed transplanted group together. No abnormal staining was found in the single nontransplanted, lesion-only control rat. 
geneic transplants, indicating that the type of transplant was not an important factor. Overall, there was a $730 \%$ increase in the number of RT 97 positive profiles in the transplanted hippocampi compared to the non-transplanted hippocampi. No abnormal RT97 staining was found in any other brain region examined.

In addition, but in contrast to the first experiment, abnormal RT97 positive profiles were found within half of the syngeneic and allogeneic transplants, as well as within the surrounding host hippocampi (Fig. 4) (nine RT97 positive perikarya and axonal swellings were found in 4 of the 8 syngeneic transplants; and 4 abnormal structures were found in 2 of the 4 allografts). However, this was only observed in those transplants which were growing as a discreet mass between the hippocampus and the stria medullaris and which appeared to be partly connected with the hippocampus and unconnected to any other brain region. No abnormal RT97 staining was found within those transplants which were placed well within the hippocampus (as with the first experiment), despite the abnormal perikaryal staining of the surrounding host tissue.

\section{DISCUSSION}

The two experiments reported here showed, firstly, that intrahippocampal cell suspension transplants of allogeneic or syngeneic tissue could survive for up to one year in fimbria-fornix lesioned rats, without evidence of immunological rejection of allogeneic transplants; and secondly that, despite this good survival of the transplants, neuropathological damage to the host hippocampal cells surrounding the transplants of either type had occurred (in a majority of cases without similar damage to the transplants themselves). This neuropathology consisted of: (i) accumulations of phosphorylated neurofilaments (and/or ectopic phosphorylation of neurofilaments) within the affected cell bodies and axonal swellings, 11 to 12 months after transplantation, and (ii) degeneration of host CAl pyramidal cells adjacent to the transplants. The CA1 cell degeneration was only found where the transplants were located close to or adjacent to the CA1 cell layer (in 3 out of the 10 transplanted rats). Due to the length of time after transplantation (one year), it is unlikely that this damage could have been caused by the transplantation procedures per se. Instead, it may have been due to either the release of toxic substances from the transplants into the host or to the expanding growth of the transplants resulting in physical damage to the adjacent pyramidal cells and their processes.

\section{Intrahippocampal transplant survival}

All of the transplants, whether syngeneic or allogeneic, had survived within the denervated hippocampus, up to one year after grafting. The results from experiment 1 , in particular, showed that transplants of PVG allogeneic neural tissue into the denervated hippocampi can survive for up to one year without any histological evidence of immunological rejection and despite the immunisation of the host with irradiated PVG splenocytes eleven months after transplantation. In contrast, grafting of fetal PVG basal forebrain tissue into the cortex of the female F344 rats from the same litters did result in graft rejection (Patel et al., in preparation) and, interestingly, the rejection of these intracortical allografts clearly displayed a spatial gradient, with grafts located on the cortical surface showing extensive rejection (in $92 \%$ of rats) and deeper, intraparenchymal, grafts remaining relatively intact (rejection in $46 \%$ of rats). The results from both of the experiments reported here as well as other evidence /32/ suggests that the deafferented hippocampus may be a transplant site relatively well protected from host immunological responses.

\section{Host neurofilament abnormality}

The neurofilament abnormality, consisting of abnormal accumulations of phosphorylated neurofilaments within cell bodies and axonal swellings, was present in significantly greater frequency in the bilateral allogeneic transplanted rats in experiment 1 ; in the transplanted side of the unilaterally transplanted rats and in the bilateral syngeneic, allogeneic and mixed transplanted rats, compared to the non-transplanted, lesion-only control hippocampi of experiment 2 (Figs. 3,5; Table 1). An indication of the magnitude of this transplant- 
associated cytoskeletal damage is given by the finding that a maximum total of 131 RT97 positive profiles was found in the hippocampal tissue surrounding the allografts of one of the transplanted rats in 15 sections ( $=8.73$ profiles per section); by comparison, the maximum incidence in the nontransplanted hippocampi was 3 profiles in 12 sections $(=0.25$ profiles per section $)$.

The highest incidence of the RT97 positive neurofilament abnormality was found in the transplanted rats which were both tolerised and immunised although, individually, the immunological procedures of tolerisation and immunisation per se had little effect upon the host brain or upon the allografts (Table 1). However, any involvement of an immunological component that this data may indicate (e.g. formation of autoantibody) in this neuropathology does not seem likely, because the damage was restricted locally to the hippocampus. Any humoral immunological factor would be expected to have had a widespread effect upon other brain regions, and not solely upon the hippocampus. In addition, rats with syngeneic only transplants (in experiment 2) also showed this neurofilament abnormality.

Similar RT97 positive cytoskeletal abnormality has been observed previously in transplantation experiments by Doering et al. $/ 8,9 /$, but with a major difference: they found numerous abnormal RT97 positive structures only within the solid transplants placed into the denervated hippocampus, but not in the surrounding host cells. In direct contrast, our first experiment showed cytoskeletal aberrations only within the host hippocampal tissue surrounding the transplants but not of the transplanted neurons. Although in both Doering's studies and those reported here, the transplants were placed into the hippocampus of fimbria-fornix lesioned rats and were examined 12 months after transplantation, there were two main differences: Doering used solid, syngeneic transplants, whereas here, we used allogeneic, cell suspension transplants. Thus, either cell suspension transplantation per se, or the allogeneic nature of the transplanted tissue, or both, may be major aetiological factors associated with the pathogenesis of the observed host hippocampal neurofilament abnormality. Experiment 2, however, shows that the damage is not restricted to alloge- neic transplantation: syngeneic grafted rats also had this neuropathology.

The perikaryal neurofilament accumulations within transplanted neurons in Doering's experiments were shown to be the result of abnormal graft-host connections /8/. Here, in partial support of these observations, abnormal RT97 staining of transplanted cells was also found, but only of those transplants (either allogeneic or syngeneic from experiment 2) which were located outside the hippocampal neuropil and which appeared to be only partially connected to the hippocampus. However, in the rats which had transplants positioned well within the hippocampal neuropil, and thus where more appropriate grafthost connections may have been established $/ 25 /$, no abnormal cytoskeletal alterations were found within the transplanted cells themselves. But, in contrast to Doering's observations, numerous neurofilament accumulations were also present within cells of the host hippocampus surrounding these transplants, irrespective of whether the transplants were located wholly or partially within the hippocampus. These results perhaps reflect.differences between the transplantation of solid tissue (as used in Doering's experiments) and of cell suspensions (as used here). Further experiments are therefore required to understand the mechanisms by which one form of transplant appears to cause host neuropathology 12 months after transplantation and the other does not. In particular, the nature of the effect of extensive graft-host connections needs to be further addressed.

Therefore, the data suggest that transplantation of neural tissue in cell suspension form per se was a critical factor associated with the development of the neurofilament abnormality in the long term. The damage was not found to be specific to the type of transplant (syngeneic or allogeneic) and could not have been due to ageing alone or solely to the effects of the fimbria-fornix lesion. In addition, our recent investigations of one year old intracortical transplants showed no RT97 abnormality of the host cortex or of the transplants, suggesting that the hippocampus may be particularly susceptible to this form of transplant associated damage.

The RT97 antibody detects the $200 \mathrm{kDA}$ phosphorylated neurofilament protein /1/, which 
normally constitutes the side-arms of neurofilaments found within axons $/ 26 /$. The phosphorylation of the protein occurs within distal parts of the axon in the normal brain and hence the phosphorylated form of the protein is not normally present within the cell bodies of neurons. In addition to lesions of neural pathways (the "axon reaction" /20,21,28/), abnormal neurofilament accumulations within neuronal perikarya and axonal retraction balls have also been found following head trauma (or diffuse axonal injury) in man $/ 16 /$ and animals $/ 33 /$; and in experimental animals intoxicated with aluminium salts $/ 19,30 /$ or with $\beta$ - $\beta$-iminodiproprionitrile $/ 17 /$, but not with 6-hydroxydopamine $/ 20$. Furthermore, the RT97 antibody also labels identical phosphorylated epitopes on neurofibrillary tangles in Alzheimer's disease $/ 1,31 /$, Pick bodies in Pick's disease $/ 31 /$ and swollen neurons in many other neurodegenerative conditions $/ 7,14,18,24,27 /$. However, the pathogenic mechanisms by which these reactions are produced are still largely unknown.

The aberrant displacement and/or phosphorylation of neurofilaments within cell bodies and axonal spheroids appears to be a specific type of response following particular insults to the brain or a result of a nervous system disease and not a general, non-specific reaction to injury, despite its widespread occurrence under different conditions. Fimbria-fornix lesioning plus neural transplantation appear to have created the specific conditions required for the induction of the observed cytoskeletal abnormalities in the host brain.

The neurofilament abnormality was found specifically within the host hippocampus in the hippocampal area surrounding the transplants and not in any other brain region examined, indicating that it must have been due to a local effect of the transplant itself or to the release of substances by certain local immune effector cells (e.g. by microglia and macrophages). The most likely explanation for this damage is that the expanding growth of the cell suspension transplants may have led to the severance or constriction of host axons, creating a condition similar to an "axon reaction" $/ 15,20,21$, $28 /$ in the damaged nerves and cells, thereby leading to the displacement or aberrant phosphorylation of neurofilament proteins. It is also possible that the observed transplant-associated cytoskeletal abnor- mality may be an unrelated phenomenon with a different pathogenic mechanism, perhaps one which involves graft-host connectivity (as discussed above).

The neurofilament abnormality was found to varying degrees in all but one of the transplanted rats examined with RT97 immunohistochemistry (16 out of 17 rats) in two separate experiments, demonstrating that it is a replicable phenomenon in this long-term transplantation paradigm, which may be used to study further the pathogenesis of such cytoskeletal disturbances and to investigate ways to prevent it, particularly if neural transplantation is to be used for the treatment of neurodegenerative diseases in man.

\section{ACKNOWLEDGEMENTS}

The authors wish to thank Prof. Brian Anderton for the gift of the RT97 antibody. This work was supported by the U.K. Medical Research Council.

\section{REFERENCES}

1. Anderton BH, Breinberg $\mathrm{D}$, Downes MJ, Green PJ, Tomlinson BE, Ulrich J, Wood JN, Kahn J. Monoclonal antibodies show that neurofibrillary tangles and neurofilaments share antigenic determinants. Nature 1982; 298: 84-86.

2. Billingham RE, Brent L, Medawar PB. "Actively acquired tolerance" of foreign cells. Nature 1953; 172 : 603-606

3. Björklund A. Neural transplantation - an experimental tool with clinical possibilities. TINS 1991; 14(8): 319327.

4. Björklund A, Stenevi U, Schmidt RH, Dunnett SB, Gage FH. Intracerebral grafting of neuronal cell suspensions I. Introductions and general methods of preparation. Acta Physiol Scand Suppl 1983; 522: 1-7.

5. Brent L. Immunologically privileged sites. In: Johansson BB, Owman C, Widner $\mathrm{H}$, eds, Pathophysiology of the Blood-Brain Barrier. Amsterdam: Elsevier, 1990; 383-402.

6. Cox G. Neuropathological techniques. In: Bancroft, JD, Stevens A, eds, Theory and practice of histological techniques. London 1982: 345.

7. Dickson DW, Yen S-H, Suzuki KI, Davies P, Garcia $\mathrm{JH}$, Hirano A. Ballooned neurons in select neurodegenerative diseases contain phosphorylated neurofilament epitopes. Acta Neuropathol (Berl) 1986; 71: $216-223$

8. Doering LC. Appropriate target interactions prevent 
abnormal cytoskeletal changes in neurons: a study with intra-sciatic grafts of the septum and hippocampus. J Neurosci 1992; 12(9): 3399-3413.

9. Doering LC, Nilsson OG, Aguayo AJ. Abnormal perikaryal immunoreactivity to the phosphorylated heavy neurofilament unit in intracerebral basal forebrain transplants. Exp Neurol 1991; 111: 1-8.

10. Dunnett S. Cholinergic grafts, memory and ageing. TINS 1991; 14(8): 371-376.

11. El Badawi A, Shenk EA. Histochemical method for separate, consecutive and simultaneous demonstration of acetylcholinesterase and norepinephrine in cryostat sections. J Histochem Cytochem 1967; 15: 580-588.

12. Feldon J, Rawlins JNP, Gray JA. Fornix-fimbria section and the partial reinforcement extinction effect. Exp Brain Res 1985; 58: 435-439.

13. Freed WJ, Dymecki J, Poltorak M, Rodgers CR. Intraventricular brain allografts and xenografts: studies of survival and rejection with and without systemic sensitisation. In: Gash DM, Sladek JR Jr, eds. Prog in Brain Res. Amsterdam: Elsevier 1988; 78: 233-241.

14. Goldman JE, Yen S-H. Cytoskeletal protein abnormalities in neurodegenerative diseases. Ann Neurol 1986; 19: 209-223.

15. Goldstein ME, Cooper HS, Bruce J, Garden MJ, Lee VMY, Schlaepfer WW. Phosphorylation of neurofilament proteins and chromatolysis following transection of rat sciatic nerve. J Neurosci 1987; 7 : 1586-1594.

16. Grady MS, McLaughlin MR, Christman CW, Vladka $\mathrm{AB}$, Fligner CL, Povlishock JT. The use of antibodies targeted against the neurofilament subunits for the detection of diffuse axonal injury in humans. J Neuropath Exp Neurol 1993; 52(2): 143-152.

17. Griffin JW, Hoffman PN, Clark AW, Carroll PT, Price DL. Slow axonal transport of neurofilament proteins: impairment of $\beta$ - $\beta$ '-iminoproprionitrile administration. Science 1978; 202: 633-635.

18. Itoh T, Sobue G, Ken E, Mitsuma T, Takahashi A, Tojanowski JQ. Phosphorylated high molecular weight neurofilament protein in the peripheral motor, sensory and sympathetic neuronal perikarya: system-dependent normal variations and changes in amyotrophic lateral sclerosis and multiple system atrophy. Acta Neuropathol 1992; 83: 240-245.

19. Johnson GVW, Jope RS. Phosphorylation of rat brain cytoskeletal proteins is increased after orally administered aluminium. Brain Res 1988; 456: 95103.

20. Klosen P, Anderton BH, Brion J-P, van den Bosch de Aguilar P. Perikaryal neurofilament phosphorylation in axotomized and 6-OH-dopamine-lesioned CNS neurons. Brain Res 1990; 526: 259-269.

21. Koliatsos VE, Applegate MD, Kitt CA, Walker LC, Delong MR, Price DL. Aberrant phosphorylation of neurofilaments accompanies transmitter-related changes in rat septal neurons following transection of the fimbria-fornix. Brain Res 1989; 482: 205-218.

22. Lindvall $O$. Prospects of transplantation in human neurodegenerative diseases. TINS 1991; 14(8): 376384.

23. Mason DW, Charlton HM, Jones AJ. The fate of allogeneic and xenogeneic neuronal tissue transplanted into the third ventricle of rodents. Neuroscience 1986; 19: 685-694.

24. Nakazato Y, Hirato J, Ishida Y, Hoshi S, Hasegawa M, Fukuda T. Swollen cortical neurons in CreutzfeldtJakob disease contain a phosphorylated neurofilament epitope. J Neuropath Exp Neurol 1990; 49(3): 197205.

25. Nilsson OG, Clarke DJ, Brundin P, Björklund A. Comparison of growth and reinnervation properties of cholinergic neurons from different brain regions grafted to the hippocampus. J Comp Neurol 1988; 268: 204-222.

26. Nixon RA. The regulation of neurofilament protein dynamics by phosphorylation: clues to neurofibrillary pathobiology. Brain Pathology 1993; 3: 29-38.

27. Pietrini V, Danielli D, Bevilacqua P, Lechi A. Panencephalopathic type of Creutzfeldt-Jakob disease with neuropathologic features similar to Pick's disease. Cllin Neuropathol 1993; 12(1): 1-7.

28. Rosenfeld J, Dorman ME, Griffin JW, Sternberger LA, Sternberger $\mathrm{NH}$, Price DL. Distribution of neurofilament antigens after axonal injury. J Neuropathol Exp Neurol 1987; 46(1): 269-282.

29. Sinden JD, Patel SN, Hodges H. Neural transplantation: problems and prospects for therapeutic application. Current Opin in Neurol and Neurosurg 1992; 5(6): 902-908.

30. Troncoso JC, Sternberger NH, Sternberger LA, Hoffman PN, Price DL. Immunocytochemical studies of neurofilament antigens in the neurofibrillary pathology induced by aluminium. Brain Res 1986; 364: 295-300.

31. Ulrich J, Haugh $M$, Anderton BH, Probst A, Lautenschlauger C, His B. Alzheimer dementia and Pick's disease: neurofibrillary tangles and Pick bodies are associated with identical phosphorylated neurofilament epitopes. Acta Neuropathol (Berl) 1987; 73: 240-246.

32. Widner $H$, Brundin P. Immunological aspects of grafting in the mammalian central nervous system: a review and speculative synthesis. Brain Res Rev 1988; 13: $287-324$

33. Yaghmai A, Povlishock J. Traumatically induced reactive change as visualised through the use of monoclonal antibodies targeted to neurofilament subunits. J Neuropathol Exp Neurol 1992; 51(2): 158176 

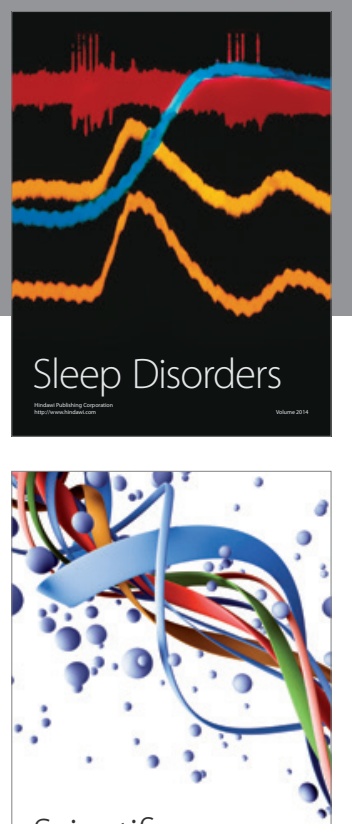

Scientifica
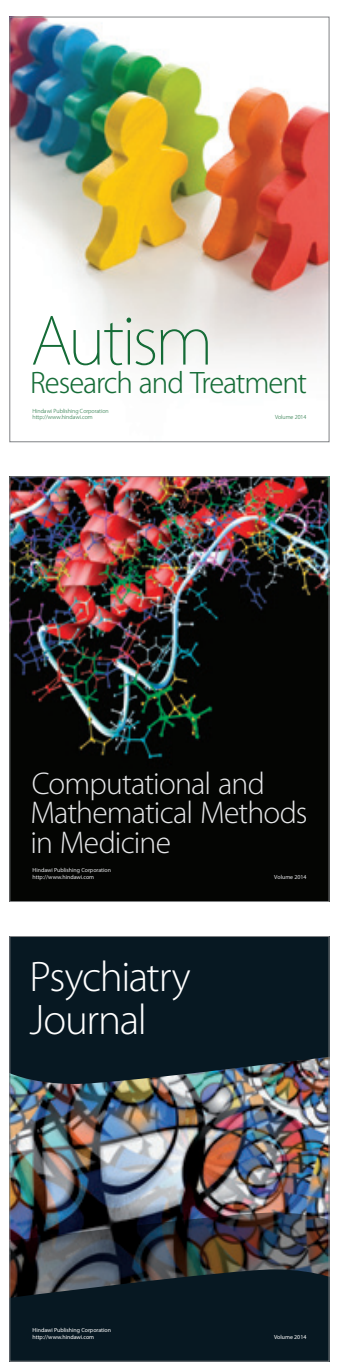
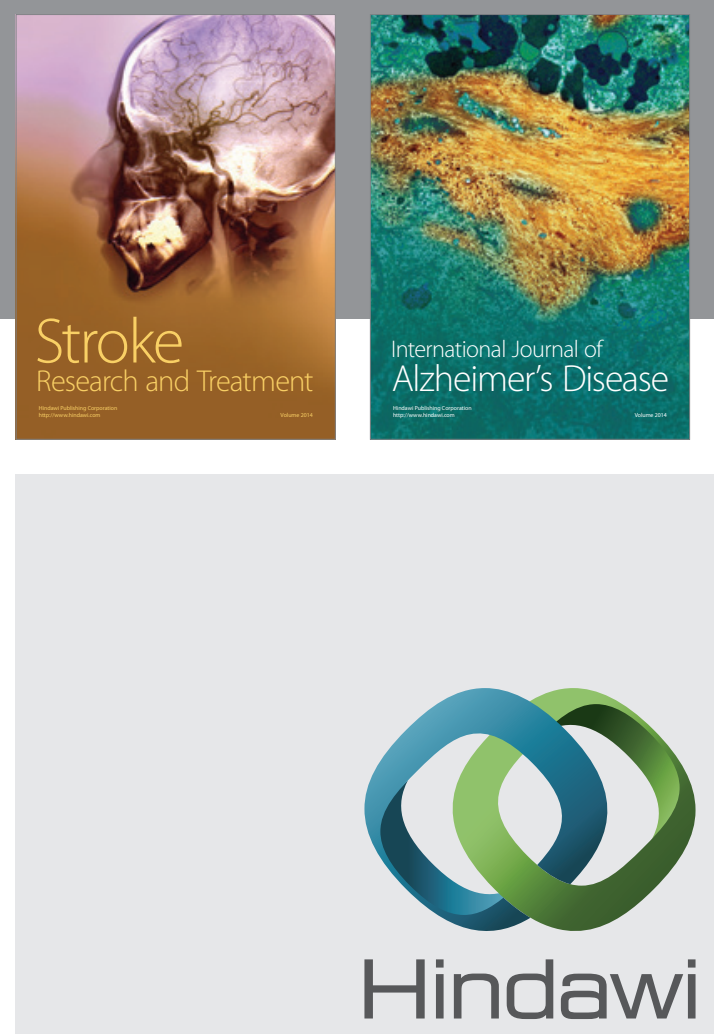

Submit your manuscripts at

http://www.hindawi.com
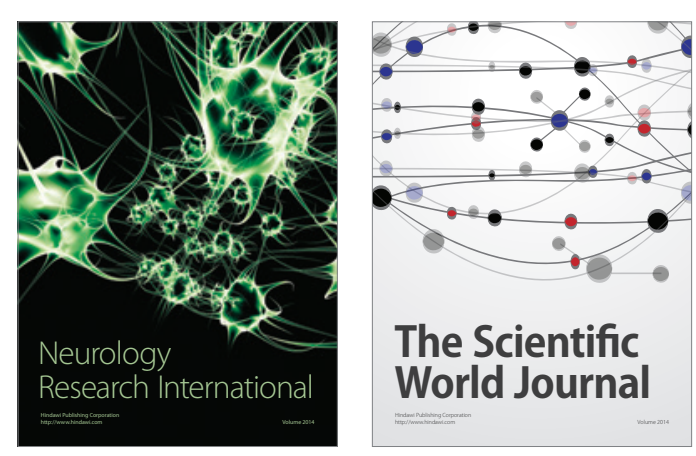

The Scientific World Journal

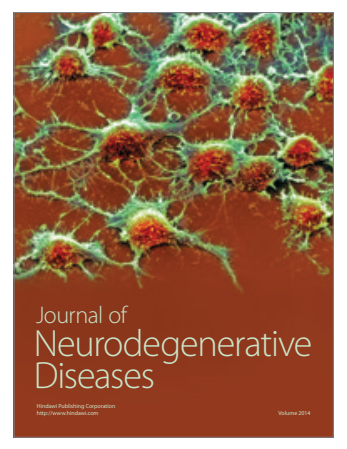

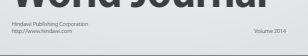

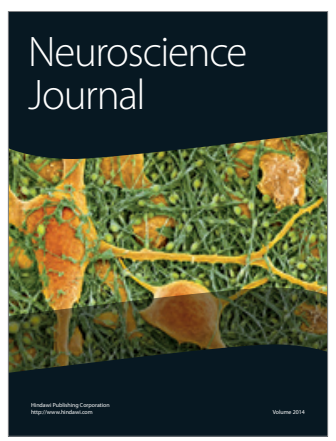

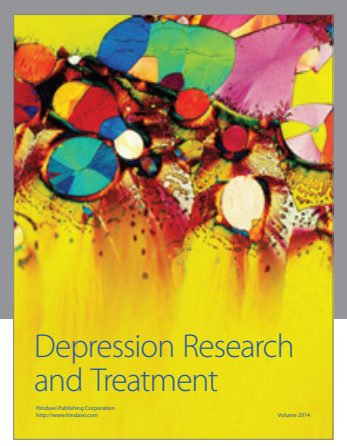
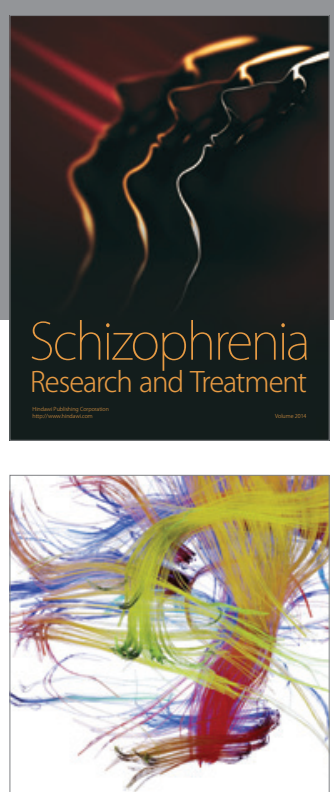

Brain Science

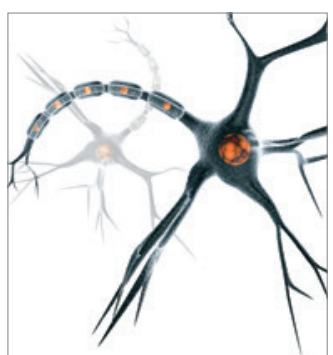

Neural Plasticity
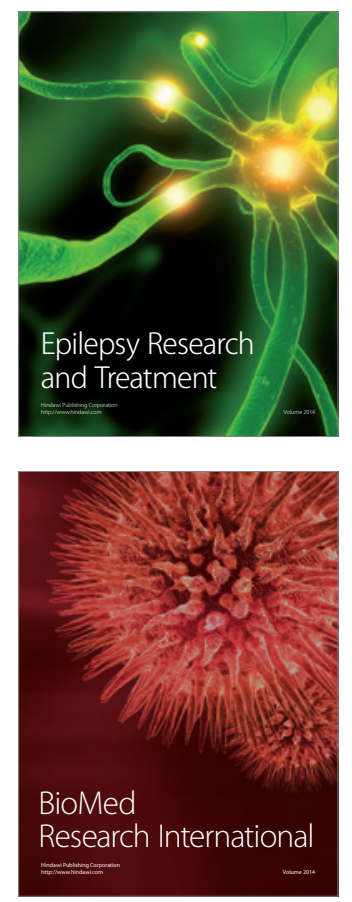

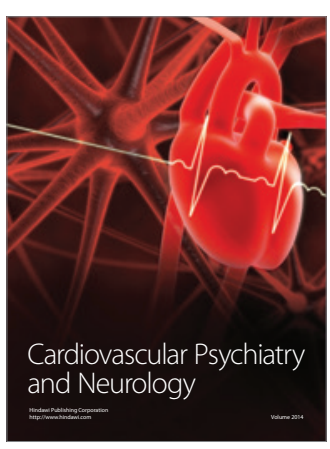

Parkinson's

Disease
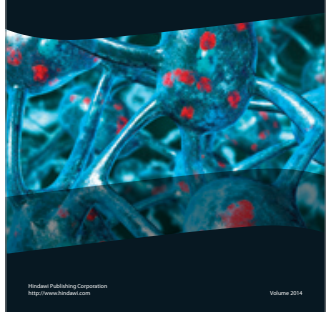